\title{
Correlação entre os achados do teste de emissão de ar nasal e da nasofaringoscopia em pacientes com fissura labiopalatina operada
}

\author{
Correlation between the findings on the nasal air emission test and \\ nasopharyngoscopy in patients with operated cleft lip and palate
}

\author{
Fabiana Andrade Penido ${ }^{1}$, Renata Matias da Silva Noronha ${ }^{2}$, Kisy Ingrid Caetano ${ }^{3}$, Marisa de Sousa Viana Jesus ${ }^{4}$, \\ Camila Queiroz de Moraes Silveira Di Ninno ${ }^{5}$, Ana Teresa Brandão de Oliveira Britto ${ }^{6}$
}

\begin{abstract}
RESUMO
Objetivo: Verificar se as informações colhidas no teste de emissão de ar nasal apresentam compatibilidade com os achados da nasofaringoscopia, podendo auxiliar no julgamento da funcionalidade do mecanismo velofaríngeo. Métodos: Foram analisados os achados do teste de emissão de ar nasal e nasofaringoscopia em 21 indivíduos operados de fissura pós-forame e transforame incisivo, de ambos os gêneros, com idade superior a oito anos. A correlação foi feita observando se, diante de um gap velofaríngeo (espaço entre as estruturas da região velofaríngea) visualizado na nasofaringoscopia, notava-se embaçamento do espelho de Glatzel no teste de emissão de ar nasal. Os dados foram analisados estatisticamente pelo índice de Kappa. Resultados: Notou-se concordância entre os testes $(\mathrm{p}<0,05)$, sendo esta considerada mais alta durante o sopro, emissão de plosivos isolados e fricativos em palavras e frases (Kappa: 0,897; 0,887; 0,774 e 0,774, respectivamente), do que para a emissão de plosivos em palavras, fricativos isolados e plosivos em frases (Kappa: 0,691; 0,640 e 0,488, respectivamente). A incompatibilidade ocorreu em poucos casos, na presença de gap pequeno, fechamento total e fechamento total com borbulha. Quanto à tendência de fechamento velofaríngeo, verificou-se predominância do tipo coronal (48\%), sendo notado melhora do mesmo com o uso de prova terapêutica em $62 \%$ dos sujeitos. Conclusão: Foi observada a validade do teste de emissão de ar nasal quando comparado à nasofaringoscopia. Contudo, é importante que o teste de emissão de ar nasal seja aplicado de forma criteriosa e que a realização deste, não substitua a nasofaringoscopia, que é conclusiva para o planejamento cirúrgico e terapêutico.
\end{abstract}

Descritores: Fissura palatina/cirurgia; Endoscopia/métodos; Insuficiência velofaríngea/diagnóstico; Fonoterapia

\section{INTRODUÇÃO}

As fissuras labiopalatinas são malformações congênitas que podem acometer o lábio (pré-forame), o palato (pós-

(1) Especialista em Motricidade Orofacial pela Pontifícia Universidade Católica de Minas Gerais - PUC-Minas - Belo Horizonte (MG), Brasil; Fonoaudióloga Clínica da Associação de Pais e Amigos do Excepcional de Mateus Leme - APAE - Mateus Leme (MG), Brasil.

(2) Especialista em Motricidade Orofacial pela Pontifícia Universidade Católica de Minas Gerais - PUC-Minas - Belo Horizonte (MG), Brasil; Fonoaudióloga Clínica do Projeto Assistencial Novo Céu e da Associação Eldorado de Apoio à Vida - Contagem (MG), Brasil.

(3) Especialista em Motricidade Orofacial pela Pontifícia Universidade Católica de Minas Gerais - PUC-Minas - Belo Horizonte (MG), Brasil; Fonoaudióloga Clínica da Prefeitura de São Sebastião do Oeste - São Sebastião do Oeste (MG), Brasil.

(4) Pós-graduanda em Lingüística pela Universidade Federal de Minas Gerais - UFMG - Belo Horizonte (MG), Brasil; Docente do Curso de Fonoaudiologia da Pontifícia Universidade Católica de Minas Gerais - PUC-Minas - Belo Horizonte (MG), Brasil; Fonoaudióloga do Centro de Tratamento e Reabilitação de Fissuras Labiopalatais e Deformidades Craniofaciais CENTRARE - Belo Horizonte (MG), Brasil. forame) ou ambos (transforame) e estar também associadas a outras malformações mais complexas, envolvendo síndromes ${ }^{(1)}$.

Os indivíduos acometidos pela fissura de palato podem

(5) Pós-graduanda em Lingüística pela Universidade Federal de Minas Gerais - UFMG - Belo Horizonte (MG), Brasil; Docente do Curso de Fonoaudiologia e Coordenadora do Curso de Especialização em Motricidade Orofacial da Pontifícia Universidade Católica de Minas Gerais - PUC-Minas - Belo Horizonte (MG), Brasil; Responsável pelo setor de Fonoaudiologia do Centro de Tratamento e Reabilitação de Fissuras Labiopalatais e Deformidades Craniofaciais - CENTRARE - Belo Horizonte (MG), Brasil.

(6) Pós-graduanda em Lingüística pela Pontifícia Universidade Católica de Minas Gerais - PUC-Minas - Belo Horizonte (MG), Brasil; Coordenadora e Professora assistente do Curso de Fonoaudiologia e Professora do Curso de Especialização em Motricidade Orofacial da Pontifícia Universidade Católica de Minas Gerais - PUC-Minas - Belo Horizonte (MG), Brasil.

Trabalho desenvolvido na Pontifícia Universidade Católica de Minas Gerais - PUC-Minas - Belo Horizonte (MG), Brasil.

Endereço para correspondência: Fabiana Andrade Penido. R. Domingos Sávio, 90/101, Bairro Santa Efigênia, Belo Horizonte - MG, CEP 30260260. E-mail: fabianafonoaudiologia@yahoo.com.br

Recebido em: 24/10/2006; Aceito em: 15/2/2007 
apresentar distúrbios articulatórios de desenvolvimento, compensatórios e/ ou obrigatórios. Os distúrbios articulatórios de desenvolvimento (linguagem e fala) são aqueles que podem estar presentes em qualquer criança e não apresentam relação direta com a fissura. Os distúrbios articulatórios compensatórios (DAC's) são provenientes de tentativas dos fissurados em compensar a válvula velofaríngeana deficiente, nas fases iniciais da aquisição da linguagem. A inadequação dos órgãos fonoarticulatórios faz com que estes indivíduos busquem outros locais de produção dos sons plosivos e fricativos, numa tentativa de bloquear a corrente de ar fonatória. Os DAC's são classificados como: golpe de glote, fricativa faríngea, plosiva dorso médio palatal, plosiva faríngea, fricativa nasal posterior e fricativa velar. Os distúrbios articulatórios obrigatórios resultam diretamente de uma alteração anatômica não sendo facilmente corrigidos com terapia e, freqüentemente, auto-corrigíveis quando a causa estrutural do distúrbio é reparada. Os distúrbios obrigatórios compreendem: a emissão de ar nasal e a pressão intra-oral reduzida, relacionados à insuficiência velofaríngea ou à presença de fístulas ${ }^{(2)}$.

$\mathrm{Na}$ fissura de palato operada, o fechamento velofaríngeo (FVF) pode se encontrar alterado quando o palato não faz um contato eficaz com as paredes da faringe e este não fechamento denomina-se disfunção velofaríngea (DVF). Esta pode estar presente em decorrência da falta de tecido na região (insuficiência velofaríngea) ou de uma inabilidade de movimentação dos músculos envolvidos no FVF (incompetência velofaríngea) $)^{(3-5)}$.

O funcionamento adequado do mecanismo velofaríngeo requer o movimento para cima e para trás do palato mole, concomitantemente ao movimento mesial das paredes laterais da faringe e ao movimento anterior da parede posterior, de forma que resulte em um $\mathrm{FVF}^{(6)}$.

Existem quatro padrões básicos de FVF: a) coronal: no qual o fechamento ocorre principalmente à custa da movimentação do véu palatino em direção à parede posterior da faringe; b) sagital ou longitudinal: quando há predominância de movimentação medial das paredes laterais da faringe em relação à do véu; c) circular: quando há movimentação equilibrada do véu e das paredes laterais da faringe; d) circular com prega de Passavant: quando a movimentação do véu, paredes laterais e parede posterior da faringe se dá de forma equilibrada $^{(7)}$.

Para se obter um equilíbrio na ressonância oro-nasal e pressão aérea intra-oral adequada durante a fala é necessário que o FVF ocorra de forma efetiva. Esse fechamento é essencial na separação da cavidade nasal e oral durante atividades pneumáticas como as de fala, sopro e assobio e atividades não pneumáticas como sucção, deglutição e reflexo de vômito. Em relação às atividades pneumáticas e não pneumáticas, existe uma hierarquia de FVF que se inicia com a deglutição, na qual este fechamento é maior, e decresce com o sopro, emissão de consoantes e, finalmente, emissão de $\operatorname{vogais}^{(8-10)}$.

A presença da DVF pode ser diagnosticada por meio da avaliação clínica da fala realizada pelo fonoaudiólogo e avaliação instrumental da função velofaríngea. A realização des- tas avaliações possibilita a definição do tratamento mais adequado para o paciente (cirurgia, prótese de palato, fonoterapia ou a combinação destes) ${ }^{(11)}$.

A avaliação clínica da fala envolve aspectos de articulação e ressonância. Para avaliar a articulação, o fonoaudiólogo observa as estruturas fonoarticulatórias (bochechas, lábios, língua, arcada dentária e palato), presença de DAC's, de fístulas e/ou fissura ainda não reparada. Este profissional ainda verifica a ocorrência de emissão de ar nasal, fraca pressão intra-oral e mímica facial associada à produção de cada fonema testado, pois estes sintomas são característicos da DVF. A ressonância é avaliada pela observação da fala espontânea e repetição de vocábulos e frases, permitindo desta forma uma análise indireta da função velofaríngea ${ }^{(12)}$.

A ressonância é classificada em: equilíbrio oro-nasal; hipernasalidade leve, moderada ou grave; hiponasalidade leve, moderada ou grave, além de ressonância mista. A hipernasalidade é o tipo mais freqüente de alteração da ressonância, observada em pacientes com fissura labiopalatina, especialmente naqueles que apresentam $\mathrm{DVF}^{(11)}$. Muitos fatores podem contribuir para a hipernasalidade, como o grau de abertura velofaríngea, a presença de obstrução nasal, o grau de abertura ou movimentação mandibular e postura elevada da língua ${ }^{(13)}$. É importante saber que não há uma relação direta entre o grau de hipernasalidade e o grau de abertura velofaríngea ${ }^{(13-14)}$.

Ainda, como parte da avaliação clínica, o fonoaudiólogo realiza o teste de emissão de ar nasal (TEAN), por meio da utilização do espelho nasal milimetrado de Altmann (ENMA) que também é conhecido como espelho de Glatzel. Para que se obtenha um resultado confiável, é importante que o espelho seja resfriado, o que marca bem a diferença de temperatura entre o ar que sai das narinas e a superfície do espelho. Além disso, deve-se assegurar que o paciente não esteja apresentando congestão nasal e, caso este possua uma fístula de palato, o teste deve ser realizado com a fístula aberta e vedada, podendo-se utilizar retalho de hóstia para este fim. Esse cuidado é necessário para que seja descartada a influência da fístula na avaliação do fechamento velofaríngeo, uma vez que o embaçamento visto no espelho pode ser decorrente do fluxo aéreo pela fístula e não pela velofaringe ${ }^{(14-17)}$.

A avaliação clínica permite que se façam hipóteses a respeito da função velofaríngea do paciente, contudo não se deve descartar a importância da avaliação instrumental, pois esta permite a determinação da causa, do grau e a localização da $\mathrm{DVF}^{(14)}$.

Existem vários exames diretos que permitem a visualização das estruturas da região velofaríngea, tanto em situação de repouso quanto durante o desenvolvimento de atividades motoras como a fala. Entre os métodos diretos, encontram-se a nasofaringoscopia, a videofluoroscopia, a radiografia lateral da cabeça, a tomografia computadorizada, a ressonância magnética e a ultra-sonografia ${ }^{(14,18)}$.

A nasofaringoscopia, atualmente, é um dos exames mais empregados no diagnóstico da DVF e acompanhamento de resultados cirúrgicos e terapêuticos desses casos. O nasofaringoscópio flexível consiste em um tubo longo, que contém fibras óticas, cujo diâmetro varia de dois a quatro 
mm. Em uma das extremidades, existe uma lente, que é utilizada para captar a imagem e, na outra extremidade, uma microcâmera de vídeo para gravação de imagens. O sistema completo inclui, ainda, uma fonte de luz fria, um videocassete, uma impressora para fotografias, um monitor de TV e uma cadeira ${ }^{(14,17)}$.

A nasofaringoscopia deve ser realizada em conjunto pelo médico e pelo fonoaudiólogo, cabendo ao último avaliar os aspectos funcionais das estruturas velofaríngeas e relacionálas com as manifestações clínicas de fala e ressonância. O nasofaringoscópio flexível é inserido na narina do paciente, preferencialmente pelo meato médio. $\mathrm{O}$ exame é iniciado pela observação da anatomia das estruturas em repouso, simetria do palato mole e paredes laterais da faringe. Em seguida, avalia-se o funcionamento destas estruturas durante a fala (emissão das vogais /a/ e /i/, emissão prolongada das fricativas não vozeadas e emissão de vocábulos e frases com fonemas plosivos e fricativos, sopro e sopro com projeção de língua) onde é possível visualizar a mobilidade de palato mole, paredes laterais e posterior da faringe, tipo de fechamento velofaríngeo (coronal, sagital, sagital com prega de Passavant e circular) e presença de gap velofaríngeo ${ }^{(14,19-20)}$. Este exame é um método de avaliação direta muito útil, pois possibilita a observação dos aspectos anatômicos e fisiológicos que causam a disfunção velofaríngea, além de auxiliar na definição do tratamento apropriado, seja ele cirúrgico, terapêutico e ou protético ${ }^{(14)}$.

A despeito do uso freqüente do TEAN na avaliação fonoaudiológica clínica e da nasofaringoscopia, sendo usados ora em conjunto, ora isolados, na bibliografia consultada não foram encontrados estudos que correlacionassem os achados destes testes no que concerne à função velofaríngea em indivíduos com fissura labiopalatina. Verificar a efetividade do TEAN, isto é, sua correspondência com o que é observado na avaliação direta, por meio da nasofaringoscopia, ou suas limitações pode permitir uma postura mais adequada do avaliador em relação ao raciocínio diagnóstico e decisões a serem tomadas quanto à conduta terapêutica quando esse dispuser somente de uma ou ambas as avaliações.

Diante de tal necessidade, o presente estudo teve como objetivo verificar se o resultado do teste de emissão de ar nasal apresenta compatibilidade com os achados da nasofaringoscopia, podendo auxiliar no julgamento da funcionalidade do mecanismo velofaríngeo. Assim, buscou-se:

- Analisar se diante de um gap velofaríngeo observado na nasofaringoscopia, nota-se no TEAN o embaçamento do espelho;

- Identificar se a produção de determinados fonemas propicia maior ou menor correlação entre os dois testes;

- Verificar o tipo de fechamento velofaríngeo (coronal, circular, circular com prega de Passavant e sagital) mais freqüente nessa população.

\section{MÉTODOS}

O presente estudo foi aprovado pelo Comitê de Ética em Pesquisa da Pontifícia Universidade Católica de Minas Gerais sob o número CAAE 0103.0.213.000-05.
Os sujeitos desta pesquisa correspondem aos indivíduos portadores de fissura pós-forame ou transforame incisivo operada, atendidos no Centro Clínico de Fonoaudiologia da PUC Minas e no CENTRARE (Centro de Tratamento e Reabilitação de Fissuras Labiopalatais e Deformidades Craniofaciais - Hospital da Baleia - $\mathrm{BH}$ ) que foram submetidos ao teste de emissão de ar nasal e nasofaringoscopia para diagnóstico da função velofaríngea e definição de conduta terapêutica.

Foram realizadas 34 avaliações, no período de agosto de 2005 a abril de 2006, sendo que deste número apenas 21 foram analisadas. Como critério de inclusão, foram considerados indivíduos dos dois sexos, com idade superior a oito anos. Os fatores de exclusão envolveram a presença de distúrbios articulatórios compensatórios em fala dirigida (DAC's), retalho faríngeo, alterações cognitivas, síndromes, fístulas que impossibilitassem seu vedamento e obstrução de vias aéreas superiores (VAS) que impedissem a realização dos testes.

Dentre os sujeitos avaliados, 11 eram do sexo feminino e 10 do sexo masculino. A idade variou entre oito e 34 anos com faixa etária média de 17 anos. Desse total, 11 apresentavam fissura transforame incisivo, seis fissura pós-forame completa, três fissura pós-forame incompleta e um, fissura submucosa.

O TEAN e a nasofaringoscopia foram realizados no Centro Clínico de Fonoaudiologia da PUC Minas. Para garantir o julgamento dos dados e a fidedignidade dos resultados, as avaliações foram desenvolvidas por duas fonoaudiólogas e um otorrinolaringologista, experientes na avaliação da função velofaríngea. Estes profissionais acompanharam todos os exames.

A avaliação fonoaudiológica foi realizada por meio do teste de emissão de ar nasal com o paciente sentado. O espelho de Glatzel foi posicionado embaixo das narinas do paciente, após este assoar as narinas, a fim de verificar a presença de escape de ar nasal durante o sopro e a produção dos sons orais da fala. A análise do TEAN foi considerada (+) diante do embaçamento do espelho e (-) na ausência desse embaçamento (Anexo 1).

Posteriormente, os sujeitos foram submetidos à avaliação otorrinolaringológica que envolveu uma observação clínica da cavidade nasal e orofaringe, por meio da realização da nasofaringoscopia flexível (Nasofibroscópio Machida $\varnothing$ 3,2 mm; câmera; fonte de luz Sigmed 250 watts; videocassete Panasonic \& Head HI-FI Stereo; fita para videocassete Niponic T120; monitor de vídeo Sony; telescópio de laringe $30^{\circ}$ llo). O esfíncter velofaríngeo foi avaliado em repouso, durante o sopro e a fala. No momento da avaliação foram observados presença ou ausência de fechamento velofaríngeo; o tipo de fechamento; tamanho do gap; mobilidade de palato mole e paredes laterais da faringe e o comportamento da região velofaríngea no momento da produção do sopro, plosivos e fricativos isolados e vocábulos (Anexo 1).

A nasofaringoscopia permitiu classificar o tipo de tentativa de fechamento do mecanismo velofaríngeo, sendo esses classificados em coronal, circular, circular com prega de Passavant e sagital ${ }^{(7)}$. Diz-se "tentativa ou tendência de fe- 
chamento" por considerar que nos casos estudados, os sujeitos apresentam inadequação velofaríngea e esse fechamento nem sempre ocorria.

Para designar o tamanho do gap, ao exame nasofaringoscópico, foram consideradas cinco categorias: ausência de gap ou fechamento velofaríngeo total $(\mathrm{Ft})$; fechamento velofaríngeo total com borbulhas (Ftb), quando não se visualizava o gap, mas notava-se escape de ar uma vez que se viam borbulhas durante a fala; gap pequeno $(\mathrm{Gp})$; médio (Gm); ou gap grande (Gg). A definição dos tamanhos do gap não foi submetida à quantificação numérica, sendo realizada uma avaliação subjetiva, na qual três avaliadores chegavam a um consenso em relação a este tamanho.

Após o término das avaliações foi realizada a correlação entre os achados do teste de emissão de ar nasal e nasofaringoscopia, utilizando o teste estatístico Kappa.

\section{RESULTADOS}

Todos os sujeitos realizaram o TEAN durante o sopro, emissão de fonemas fricativos e plosivos isolados, em palavras e frases. Essas mesmas emissões foram realizadas, utilizando-se a nasofaringoscopia. Tais resultados podem ser vistos na Tabela 1 .

Com vista a analisar se havia concordância entre os dois exames, utilizou-se o teste estatístico Kappa, cujo resultado pode ser observado na Tabela 2 .

Observou-se compatibilidade entre os testes em 20 (95\%) sujeitos durante o sopro. Na produção de plosivos, notou-se compatibilidade em $20(95 \%)$ deles durante emissão isola-

Tabela 1. Achados do teste de emissão de ar nasal e nasofaringoscopia, durante a produção do sopro, emissão de plosivos e fricativos isolados, em palavras e frases, para cada sujeito $(n=21)$

\begin{tabular}{|c|c|c|c|c|c|c|c|c|}
\hline Sujeitos & Exames & Sopro & $\begin{array}{l}\text { Plosivo } \\
\text { isolado }\end{array}$ & $\begin{array}{l}\text { Plosivo } \\
\text { palavra }\end{array}$ & $\begin{array}{l}\text { Plosivo } \\
\text { frase }\end{array}$ & $\begin{array}{c}\text { Fricativo } \\
\text { isolado }\end{array}$ & $\begin{array}{c}\text { Fricativo } \\
\text { palavra }\end{array}$ & $\begin{array}{c}\text { Fricativo } \\
\text { frase }\end{array}$ \\
\hline \multirow[t]{2}{*}{1} & TEAN & - & - & - & - & - & + & + \\
\hline & NASO & $\mathrm{Ft}$ & $\mathrm{Ft}$ & Ftb & Gp & $\mathrm{Ft}$ & $\mathrm{Gm}$ & $\mathrm{Gm}$ \\
\hline \multirow[t]{2}{*}{2} & TEAN & + & + & + & + & + & + & + \\
\hline & NASO & $\mathrm{Gm}$ & $\mathrm{Gm}$ & $\mathrm{Gm}$ & $\mathrm{Gm}$ & $\mathrm{Gm}$ & $\mathrm{Gm}$ & $\mathrm{Gm}$ \\
\hline \multirow[t]{2}{*}{3} & TEAN & + & + & + & + & + & + & + \\
\hline & NASO & $\mathrm{Gg}$ & $\mathrm{Gg}$ & $\mathrm{Gg}$ & $\mathrm{Gg}$ & $\mathrm{Gg}$ & $\mathrm{Gg}$ & $\mathrm{Gg}$ \\
\hline \multirow[t]{2}{*}{4} & TEAN & + & + & + & + & + & + & + \\
\hline & NASO & $\mathrm{Gg}$ & $\mathrm{Gg}$ & $\mathrm{Gg}$ & $\mathrm{Gg}$ & $\mathrm{Gg}$ & $\mathrm{Gg}$ & $\mathrm{Gg}$ \\
\hline \multirow[t]{2}{*}{5} & TEAN & + & + & + & + & + & + & + \\
\hline & NASO & $\mathrm{Gg}$ & $\mathrm{Gg}$ & $\mathrm{Gg}$ & $\mathrm{Gg}$ & $\mathrm{Gg}$ & $\mathrm{Gg}$ & $\mathrm{Gg}$ \\
\hline \multirow[t]{2}{*}{6} & TEAN & + & + & + & + & + & + & + \\
\hline & NASO & $\mathrm{Gg}$ & $\mathrm{Gg}$ & $\mathrm{Gg}$ & $\mathrm{Gg}$ & $\mathrm{Gg}$ & $\mathrm{Gg}$ & $\mathrm{Gg}$ \\
\hline \multirow[t]{2}{*}{7} & TEAN & + & + & + & + & + & + & + \\
\hline & NASO & $\mathrm{Ft}$ & $\mathrm{Gm}$ & $\mathrm{Gm}$ & $\mathrm{Gg}$ & $\mathrm{Ft}$ & $\mathrm{Gm}$ & $\mathrm{Gg}$ \\
\hline \multirow[t]{2}{*}{8} & TEAN & - & + & + & + & + & + & + \\
\hline & NASO & $\mathrm{Ft}$ & Ftb & Ftb & Ftb & $\mathrm{Ft}$ & Ftb & Ftb \\
\hline \multirow[t]{2}{*}{9} & TEAN & - & - & - & - & - & + & + \\
\hline & NASO & $\mathrm{Ft}$ & $\mathrm{Ft}$ & $\mathrm{Gp}$ & Gp & Gp & Gp & Gp \\
\hline \multirow[t]{2}{*}{10} & TEAN & + & + & + & + & + & + & + \\
\hline & NASO & $\mathrm{Gm}$ & $\mathrm{Gm}$ & $\mathrm{Gg}$ & $\mathrm{Gg}$ & $\mathrm{Gm}$ & $\mathrm{Gg}$ & $\mathrm{Gg}$ \\
\hline \multirow[t]{2}{*}{11} & TEAN & - & - & + & + & - & + & + \\
\hline & NASO & $\mathrm{Ft}$ & $\mathrm{Ft}$ & Gp & Gp & $\mathrm{Ft}$ & Gp & $\mathrm{Gp}$ \\
\hline \multirow[t]{2}{*}{12} & TEAN & + & + & + & + & + & + & + \\
\hline & NASO & $\mathrm{Gg}$ & $\mathrm{Gg}$ & $\mathrm{Gg}$ & $\mathrm{Gg}$ & $\mathrm{Gg}$ & $\mathrm{Gg}$ & $\mathrm{Gg}$ \\
\hline \multirow[t]{2}{*}{13} & TEAN & + & + & + & + & + & + & + \\
\hline & NASO & $\mathrm{Gg}$ & $\mathrm{Gg}$ & $\mathrm{Gg}$ & $\mathrm{Gg}$ & $\mathrm{Gg}$ & $\mathrm{Gg}$ & $\mathrm{Gg}$ \\
\hline \multirow[t]{2}{*}{14} & TEAN & + & + & + & + & + & + & + \\
\hline & NASO & $\mathrm{Gg}$ & $\mathrm{Gg}$ & $\mathrm{Gg}$ & $\mathrm{Gg}$ & $\mathrm{Gg}$ & $\mathrm{Gg}$ & $\mathrm{Gg}$ \\
\hline \multirow[t]{2}{*}{15} & TEAN & + & + & + & + & + & + & + \\
\hline & NASO & $\mathrm{Gg}$ & $\mathrm{Gg}$ & $\mathrm{Gg}$ & $\mathrm{Gg}$ & $\mathrm{Gg}$ & $\mathrm{Gg}$ & $\mathrm{Gg}$ \\
\hline \multirow[t]{2}{*}{16} & TEAN & + & + & + & + & + & + & + \\
\hline & NASO & Gp & Gp & Gp & $\mathrm{Gm}$ & Gp & Gp & $\mathrm{Gm}$ \\
\hline \multirow[t]{2}{*}{17} & TEAN & - & - & - & - & - & - & - \\
\hline & NASO & $\mathrm{Ft}$ & $\mathrm{Ft}$ & $\mathrm{Ft}$ & $\mathrm{Ft}$ & $\mathrm{Ft}$ & $\mathrm{Ft}$ & $\mathrm{Ft}$ \\
\hline \multirow{2}{*}{18} & TEAN & $-\mathrm{Ft}$ & + & + & + & + & + & + \\
\hline & NASO & & Gp & Gp & $\mathrm{Gm}$ & Gp & Gp & $\mathrm{Gm}$ \\
\hline \multirow[t]{2}{*}{19} & TEAN & + & + & + & + & + & + & + \\
\hline & NASO & $\mathrm{Gm}$ & $\mathrm{Gm}$ & $\mathrm{Gm}$ & $\mathrm{Gm}$ & $\mathrm{Gm}$ & $\mathrm{Gm}$ & $\mathrm{Gm}$ \\
\hline \multirow[t]{2}{*}{20} & TEAN & + & + & + & + & + & + & + \\
\hline & NASO & $\mathrm{Gg}$ & $\mathrm{Gm}$ & $\mathrm{Gg}$ & $\mathrm{Gg}$ & $\mathrm{Gg}$ & $\mathrm{Gg}$ & $\mathrm{Gg}$ \\
\hline \multirow[t]{2}{*}{21} & TEAN & - & - & - & - & - & - & - \\
\hline & NASO & $\mathrm{Ft}$ & $\mathrm{Ft}$ & $\mathrm{Ft}$ & $\mathrm{Ft}$ & $\mathrm{Ft}$ & Ftb & Ftb \\
\hline
\end{tabular}

Legenda: TEAN: Teste de emissão de ar nasal; NASO: Nasofaringoscopia; (+) presença de escape de ar nasal; (-) ausência de escape de ar nasal; Ft: fechamento velofaríngeo total, Ftb: fechamento velofaríngeo total com borbulha; Gp: gap pequeno; Gm: gap médio; Gg: gap grande. 
Tabela 2. Concordância do resultado do teste de emissão de ar nasal e nasofaringoscopia nas diferentes emissões, para todos os sujeitos ( $\mathrm{n}=21$ )

\begin{tabular}{|c|c|c|c|c|}
\hline & Emissões & Concordância & Não concordância & Índice de Kappa \\
\hline Modo articulatório & Sopro & $20(95 \%)$ & $1(5 \%)$ & Kappa $=0,897$ \\
\hline \multirow[t]{3}{*}{ Plosivos } & Emissão isolada & 20 (95\%) & $1(5 \%)$ & Kappa $=0,887$ \\
\hline & Palavras & $19(91 \%)$ & $2(9 \%)$ & Kappa $=0,691$ \\
\hline & Frases & $18(86 \%)$ & $3(14 \%)$ & Kappa $=0,488$ \\
\hline \multirow[t]{3}{*}{ Fricativos } & Emissão isolada & $18(86 \%)$ & $3(14 \%)$ & Kappa $=0,640$ \\
\hline & Palavras & 20 (95\%) & $1(5 \%)$ & Kappa $=0,774$ \\
\hline & Frases & $20(95 \%)$ & $1(5 \%)$ & Kappa $=0,774$ \\
\hline
\end{tabular}

Índice de Kappa $(p<0,05)$

da, 19 (91\%) durante emissão de palavras e 18 (86\%) durante a emissão de frases. Os resultados com sons fricativos demonstraram concordância em $18(86 \%)$ pacientes durante emissão isolada e 20 (95\%) durante produções de palavras e frases. Dentre as incompatibilidades encontradas, notou-se a ausência de escape de ar nasal em sujeitos com gap pequeno e presença de escape de ar nasal, quando havia fechamento total e fechamento total com borbulha. A análise estatística demonstrou a existência de concordância significativa entre os testes $(\mathrm{p}<0,05)$, sendo esta considerada mais alta durante o sopro e a emissão de plosivos isolados e fricativos em palavras e frases (Kappa: 0,897; 0,887; 0,774 e 0,774, respectivamente), do que para a emissão de plosivos em palavras, fricativos isolados e plosivos em frases (Kappa: 0,691; 0,640 e 0,488 , respectivamente).

Não se notou diferenças nos resultados em decorrência do modo articulatório dos fonemas, isto é, se o som era plosivo ou fricativo. $\mathrm{O}$ mesmo ocorre quanto à extensão da emissão, se som isolado, em palavras ou frases. Ou seja, independente do tipo de emissão houve correspondência entre presença de gap velofaríngeo e embaçamento do espelho.

Em relação à tendência de fechamento velofaríngeo da amostra analisada, verificou-se predominância do tipo coronal (48\%), seguido do circular (38\%), sagital $(9,5 \%)$ e circular com prega de Passavant $(4,5 \%)$.

O protocolo de teste utilizado ainda compara a funcionalidade do mecanismo velofaríngeo com e sem o treino articulatório. Assim, após o TEAN e nasofaringoscopia, orienta-se o paciente a direcionar o fluxo aéreo para a cavidade oral, tentando fechar o mecanismo velofaríngeo com apoio visual, no vídeo. Objetiva-se com isso verificar as reais possibilidades de melhora do funcionamento desse mecanismo quando esse sujeito é orientado em como fazê-lo. Os resultados encontrados para os 21 sujeitos analisados demonstram melhora do fechamento velofaríngeo com o uso de prova terapêutica em $62 \%$ dos pacientes.

\section{DISCUSSÃO}

A utilização do espelho de Glatzel como instrumento auxiliar na avaliação do mecanismo velofaríngeo tem uma ampla aceitação devido à sua praticidade ${ }^{(5,14-15)}$. Não se tem relato de quem preconizou esse curioso método que partia do pressuposto de que, diante da dificuldade de se visualizar o real fechamento velofaríngeo, poderia se fazer inferência sobre ele verificando se estava escapando ar pelo nariz em sons orais. E que naturalmente, isso poderia ser visto colocando-se um espelho debaixo das narinas. Dessa forma, podese verificar em quais sons esse escape nasal ocorre ou não, permitindo inclusive, inferir se esse escape acontece em todos os fonemas ou somente em alguns, se o palato é incompetente ou insuficiente.

Obviamente, esse teste tem suas limitações, pois não permite visualizar o palato, fazer medidas quantitativas do tamanho do gap velofaríngeo, sua localização e formato, conforme já discutido ${ }^{(15)}$. No caso de indivíduos com fissura, nota-se com freqüência, alterações nasais como desvio de septo, hipertrofia de cornetos, pólipos ou secreções que podem mascarar os resultados. Ainda, nos casos em que se observam distúrbios articulatórios compensatórios (DAC's), por não exigirem o fechamento velofaríngeo, nota-se escape de ar, não permitindo que se avalie as reais potencialidades do palato ${ }^{(16)}$. Outras dificuldades como a temperatura do espelho, o momento de colocá-lo e retirá-lo para que a respiração fora da fala não comprometa os resultados devem ser considerados ${ }^{(15)}$.

A nasofaringoscopia, por sua vez, é um instrumento que permite uma avaliação direta do mecanismo velofaríngeo, dando condições a que se faça uma avaliação mais detalhada quanto ao tipo de fechamento, formas do gap e ainda quanto ao funcionamento desse mecanismo, nas diferentes produções de fala, informações essas essenciais para determinadas decisões terapêuticas ${ }^{(5,14)}$. Diante de um palato anatomicamente insuficiente para permitir o fechamento, a conduta deverá ser a utilização de prótese de palato ou palatoplastia secundária; já diante de um palato com limitações funcionais, isso é com condições de alcançar o fechamento total durante a produção de determinados fonemas, a fonoterapia visando estimular a função velofaríngea é indicada ${ }^{(11)}$. Tal análise requer a utilização de exames objetivos como a videofluoroscopia ou a nasofaringoscopia ${ }^{(5,14)}$. Entretanto, tais exames nem sempre estão disponíveis ao paciente $\mathrm{e}$, às vezes, não podem ser prontamente utilizados no processo terapêutico, seja por dificuldade de acesso a um Centro que realize tais avaliações, ou por limitações financeiras que inviabilizam a utilização deles ou mesmo dificuldades na realização do exame por ser invasivo.

Refletindo sobre as indicações e contra-indicações para a utilização de avaliações diretas ou indiretas, é prudente que se considere as correlações entre eles, propósito desse trabalho. 
A análise dos resultados demonstrou a confiabilidade do teste de emissão de ar nasal (TEAN), visto que houve concordância entre o mesmo quando comparado à nasofaringoscopia em diversas emissões. Nota-se que grande número dos sujeitos avaliados apresentaram gap grande e médio. Esse fato pode ter contribuído para essa concordância, uma vez que permite maior passagem de ar, facilitando a visualização no espelho de Glatzel. Estudos com foco no gap pequeno precisam ser realizados verificando se os índices de concordância se mantêm. Também estudos utilizando espelho milimetrado que permitem a mensuração do escape de ar nasal devem ser realizados de forma a comparar o tamanho do gap e a quantidade de escape aéreo nasal.

Em alguns poucos casos, observou-se comportamentos diferenciados em que a correlação entre os exames não foi identificada. No sujeito 09, identificou-se a presença de gap velofaríngeo pequeno, sendo esperado escape de ar nasal, o qual não foi visualizado. Esta incompatibilidade também foi relatada por outros autores que levantaram a hipótese de que o fechamento velofaríngeo tenha ocorrido em um nível mais inferior da região do esfíncter velofaríngeo, o qual não foi possível ser observado, dando a impressão de um gap velofaríngeo mínimo ${ }^{(20)}$. Outra hipótese para este evento é que, apesar de existir um gap pequeno, não houve quantidade suficiente de fluxo aéreo que escapasse pela cavidade nasal levando ao embaçamento do espelho de Glatzel. Há que se considerar ainda, a possibilidade do resultado negativo no TEAN ser assistemático e, no momento da Nasofaringoscopia ter se verificado um gap $^{(14)}$.

Os fonemas fricativos e plosivos constam no protocolo, por exigirem maior pressão intra-oral, incidindo neles as dificuldades articulatórias dos pacientes com fissura ${ }^{(21-24)}$. Em pacientes, com inadequação velofaríngea, o ar escapa para as cavidades nasais, dificultando a formação de pressão intraoral requerida na produção dessas consoantes ${ }^{(25)}$. A verificação, por meio da nasofaringoscopia, da existência de fechamento velofaríngeo em algum deles, auxilia no direcionamento terapêutico. Estudos mostram que diante de compensações articulatórias o movimento do mecanismo velofaríngeo é diminuído ${ }^{(16)}$. Os sujeitos desse trabalho não apresentavam compensações articulatórias e, no entanto, constata-se gap grande na maioria das emissões com esses fonemas, com tendência a manter o mesmo tamanho do gap tanto nas plosivas quanto nas fricativas. Esse é um dado interessante, na medida em que mostra que é possível a produção de sons sem compensações mesmo na presença de insuficiência ou incompetência velofaríngea.

Diante de compensações articulatórias não se justifica a utilização do TEAN para estudo da função velofaríngea, pois o escape de ar nasal é esperado. Alguns sujeitos, a despeito de apresentarem gap grande durante a emissão de alguns fonemas, conseguiam fazer o fechamento total. Esse melhor funcionamento do palato geralmente ocorria nos fonemas isolados. Quando tais sons eram inseridos em palavras e frases, obviamente com inclusão de vogais, o palato não mantinha o mesmo padrão de fechamento. A influência da qualidade da vogal no funcionamento do palato é mostrada em vários estudos, relacionando a altura da língua e o movimen- to do palato ${ }^{(26-27)}$. Parece haver consenso na literatura de que as vogais altas tendem a estar associadas à maior elevação do palato do que as vogais baixas. As vogais baixas, mesmo orais, podem exibir uma pequena abertura no fechamento velofaríngeo $^{(9)}$. Nas frases utilizadas no protocolo, as diferentes vogais ocorrem, por certo contribuindo para o gap maior visto nesta condição em relação aos sons isolados. A inclusão de emissão de vogais isoladas nesse protocolo, em estudos posteriores, pode contribuir na identificação da interferência de cada vogal para o funcionamento do palato visto nas palavras e frases.

No que diz respeito à tendência de fechamento velofaríngeo, observou-se que o tipo coronal foi o mais encontrado na população estudada seguido do circular, sagital e circular com prega de Passavant. O tipo coronal já havia sido relatado como o mais freqüente em indivíduos tanto com disfunção velofaríngea ${ }^{(28)}$ como com funcionamento velofaríngeo adequado ${ }^{(7,20,29)}$. Notou-se diferença em relação à hierarquia dos demais padrões de fechamento velofaríngeo, todavia esta diferença pode estar relacionada ao tamanho da amostra e de certa subjetividade que envolve a classificação dos mesmos ${ }^{(20)}$. Quando comparado aos dados obtidos em sujeitos normais, o tipo coronal continua sendo o mais freqüente, contudo, é proporcionalmente menor em fissurados onde há um aumento do tipo circular ou sagital, o que demonstra a maior participação das paredes laterais nesses úl$\operatorname{timos}^{(7,28)}$. Este fato faz emergir a hipótese de que o aumento da participação das paredes laterais poderia ser uma compensação pela disfunção velofaríngea, desenvolvida espontaneamente ou pela própria fonoterapia.

Em última análise, foi avaliada a melhora do fechamento velofaríngeo com treino articulatório, verificada em $62 \%$ dos sujeitos. Este dado demonstra a importância de realização do mesmo, solicitando ao paciente o aumento do fluxo aéreo oral, sempre em busca da melhor produção, que possibilita um resultado mais fidedigno sobre as reais possibilidades de fechamento velofaríngeo ${ }^{(11)}$.

Acredita-se que este estudo possa auxiliar no diagnóstico da disfunção velofaríngea, fornecendo maior confiabilidade à avaliação clínica fonoaudiológica, realizada por meio do teste de emissão de ar nasal.

Apesar de ser encontrada grande coerência entre os resultados nos testes utilizados nesta pesquisa, torna-se necessário ressaltar que a realização do TEAN deve ser de forma criteriosa, conforme sugestões descritas na bibliografia esplanada ${ }^{(15)}$.

Pretende-se dar continuidade ao estudo, ampliando a amostra e realizando a marcação do embaçamento no espelho de Glatzel, para que este dado possa ser comparado ao tamanho do gap velofaríngeo verificado na nasofaringoscopia.

\section{CONCLUSÃO}

A partir deste estudo, pôde-se notar a validade do teste de emissão de ar nasal, que apesar de ser um procedimento que não nos permite a visualização do mecanismo velofaríngeo, auxilia no julgamento da funcionalidade do mesmo, visto a existência de significativa correlação com os achados da nasofaringoscopia. 
Uma vez que o TEAN apresenta confiabilidade, não se deve pressupor que este possa ser utilizado isoladamente para o diagnóstico e definição de condutas. É fundamental a realização de exames objetivos, como a nasofaringoscopia que possibilita a observação do funcionamento da velofaringe, caracterização quanto ao tipo de fechamento velofaríngeo e tamanho do gap.

Considera-se de grande relevância a aplicação do TEAN de forma criteriosa, a fim de se documentar avaliações de forma precisa, aumentando a confiabilidade dos procedimentos fonoaudiológicos e permitindo fazer inferências sobre o diagnóstico.

\begin{abstract}
Objective: To verify whether the information collected in the nasal air emission test are compatible to the nasopharyngoscopy findings, helping the assessment of the velopharyngeal mechanism functionality. Methods: The findings of nasal air emission test and nasopharyngoscopy were analyzed in 21 individuals of both genders, over the age of 8 years, operated for post-foramen and transincisive foramen fissure. The correlation was carried out observing whether or not, in the face of a velopharyngeal gap visualized in the nasopharyngoscopy, misting of the Glatzel mirror was noted in the nasal air emission test. Data were statistically analyzed using the Kappa index. Results: An agreement was observed between the tests $(\mathrm{p}<0,05)$, and it was considered higher during blowing, emission of isolated stops and fricatives in words and phrases (Kappa: 0,897; 0,887; 0,774 and 0,774, respectively), than for emission of stops in words, isolated fricatives and stops in phrases (Kappa: 0,691; 0,640 and 0,488, respectively). Incompatibility occurred in a few cases, in the presence of a small gap, complete closing and complete closing with bubbling. Regarding the tendency of velopharyngeal closing, it was found a predominance of the coronal type (48\%), and improvement was noted in $62 \%$ of the subjects with the use of therapeutic test. Conclusion: The validity of the nasal air emission test when compared with nasopharyngoscopy was observed. It is, however, important to emphasize that the nasal air emission test must be carefully applied and it shouldn't replace the nasopharyngoscopy, which is conclusive for surgical and therapeutic planning.
\end{abstract}

Keywords: Cleft palate/surgery; Endoscopy/methods; Velopharyngeal insufficiency/diagnosis; Speech therapy

\title{
REFERÊNCIAS
}

1. Capelozza Filho L, Silva Filho OG. Fissuras lábio-palatais. In: Petrelli E, coodenador. Ortodontia para fonoaudiologia. Curitiba: Lovise; 1991. p.195-239.

2. Vicente MCZ, Buchala RG. Atualização da terminologia de distúrbios articulatórios encontrados em falantes portadores de fissura de lábio e palato. Distúrb Comun. 1991;4:147-52.

3. Amaral SA, Genaro KF. Análise da fala em indivíduos com fissura labiopalatina operada. Pró-Fono. 1996;8(1):36-46.

4. Paciello RZ, Piazentin-Penna SHA. Insuficiência velofaringeana: seguimento de um caso clínico pré e pós-faringoplastia. Distúrb Comun. 1989;3(1):33-47.

5. Williams WN, Pegoraro-Krook MI, Dutka-Souza JCR, Marino VC, Wharton PW. Nasoendoscopy: the role of the speech-language pathologist. Braz J Dysmorphol Speech-Hearing Disord. 1999;3(2):2336.

6. Flowers CR, Morris HL. Oral-pharyngeal movements during swallowing and speech. Cleft Palate J. 1973;10:181-91.

7. Skolnick ML, McCall GN, Barnes M. The sphincteric mechanism of velopharyngeal closure. Cleft Palate J. 1973; 10:286-305.

8. Peterson SJ. Velopharyngeal function: some important differences. J Speech Hear Disord. 1973;38(1):89-97.

9. Matsuya T, Miyazaki T, Yamaoka M. Fiberscopic examination of velopharyngeal closure in normal individuals. Cleft Palate J. 1974;11:286-91.

10. Miyazaki T, Matsuya T, Yamaoka M. Fiberscopic methods for assessment of velopharyngeal closure during various activities. Cleft Palate J. 1975;12:107-14

11. Pegoraro-Krook MI, Souza JCRD, Teles-Magalhães LC, Feniman MR. Intervenção fonoaudiológica na fissura palatina. In: Ferreira LP, BefiLopes DM, Limongi SCO. Tratado de fonoaudiologia. São Paulo: Roca; 2004. p. 339-455.
12. Bzoch K. Clinical assessment, evaluation, and management of 11 categorial aspects of cleft palate speech disorders. In: Bzoch KR, editor. Communicative disorders related to cleft lip and palate. 4a ed. Austin: Pro-Ed; 1997. p.261-311.

13. Warren DW, Hairfield WM, Hinton WA. The respiratory significance of the nasal grimace. ASHA. 1985;27:82-9.

14. Genaro KF, Yamashita RP, Trindade IEK. Avaliação clínica e instrumental na fissura labiopalatina. In: Ferreira LP, Befi-Lopes DM, Limongi SCO, organizadores. Tratado de fonoaudiologia. São Paulo: Roca; 2004. p. 456-77.

15. Altmann EBC, Khoury RBF. Avaliação fonoaudiológica. In: Altmann EBC. Fissuras labiopalatinas. Barueri: Pró-Fono; 1992. p.313-47.

16. Henningsson GE, Isberg AM. Velopharyngeal movement patterns in patients alternating between oral and glottal articulation: a clinical and cineradiographical study. Cleft Palate J. 1986;23(1):1-9.

17. Di Ninno CQMS. Di Ninno CQMS. A Importância do vedamento da fístula de palato durante a avaliação da fala e da função velofaríngea. 2003. In: 2o. Smile Train Latin American Symposium on Cleft Lip and Palate; 2003.

18. Golding-Kushner KJ, Argamaso RV, Cotton RT, Grames LM, Henningsson $\mathrm{G}$, Jones DL, et al. Standardization for the reporting of nasopharyngoscopy and multiview videofluoroscopy: a report from an International Working Group. Cleft Palate J. 1990;27(4):337-47; discussion 347-8.

19. Kummer AW, Briggs M, Lee L. The relationship between the characteristics of speech and velopharyngeal gap size. Cleft Palate Craniofac J. 2003;40(6):590-6.

20. Camargo LOS, Rodrigues CM, Avelar JA. Oclusão velofaríngea em indivíduos submetidos à nasoendoscopia na Clínica de Educação para Saúde (CEPS). Salusvita. 2001;20(1):35-48.

21. Eckelmann D, Baldridge P. Speech training for a child with clef palate. J Speech Dis. 1945;10:137-49. 
22. Spriesterbach DC, Powers GR. Articulation skills, velopharyngeal closure, and oral breath pressure of children with cleft palates. J Speech Hear Res. 1959;2:318-25.

23. Van Demark DR. Misarticulations and listener judgements of the speech individuals with cleft palates. Cleft Palate J. 1964;1(2):232-45.

24. Trost JE. Articulatory additions to the classical description of the speech of persons with cleft palate. Cleft Palate J. 1981;18(3):193-203.

25. Witzel MA. Communicative impairment associated with clefting. In: Shprintzen RJ, Bardach J, editors. Cleft palate speech management: a multidisciplinary approach. St. Louis: Mosby; c1995. p.137-66.

26. Moon J, Kuehn D. Velopharyngeal closure force and levator veli palatini activation levels in relation to varying phonetic contexts. In: National Center for Voice and Speech Status and Progress Report. 1997;11:107-18.
27. Bell-Berti F. Understanding velic motor control: studies of segmental context. In: Huffman MK, Krakow RA, editors. Phonetics and phonology: nasals, nasalization, and the velum. San Diego: Academic Press; 1993. p. 63-85.

28. Croft CB, Shprintzen RJ, Rakoff SJ. Patterns of velopharyngeal valving in normal and cleft palate subjects: a multi-view videofluoroscopic and nasoendoscopic study. Laryngoscope. 1981;91(2):265-71.

29. Altmann EBC, Pontes PAL, Behlau MS. Normatização do exame nasolaringoscópico para o estudo das alterações do esfíncter velofaríngico. Distúrb Comun. 1990;3:239-47.

Anexo 1. Protocolo de avaliação da função velofaríngea

Avaliação da função velofaríngea

Prontuário:

Data:
1. Identificação

Nome:

DN: 1 1 Idade: Tipo de fissura:

\section{Avaliação}

\section{Condição atual do palato:}
$\square$ Íntegro
$\square$ Aberto
$\square$ Operado
$\square$ Com fístula
$\square$ Submucosa

Permeabilidade das narinas ao espelho:
D: $\square$ Permeável
$\square$ Parcialmente obstruída
$\square$ Obstruída
E: $\square$ Permeável
$\square$ Parcialmente obstruída
$\square$ Obstruída

\section{Teste de Emissão de Ar Nasal (Espelho de Glatzel):}

$\begin{array}{lll}\text { Sopro: } & \square+ \\ \text { /p/ } & \square+ & \square- \\ \text { /t/ } & \square+ & \square- \\ \text { /k/ } & \square+ & \square- \\ \text { /f/ } & \square+ & \square- \\ \text { /s/ } & \square+ & \square- \\ \text { /s/ } & \square+ & \square- \\ \text { Papaipediupipoca } & \end{array}$

Papai pediu pipoca $\quad \square+$

O tatu tá na toca

Cacá quer coca-cola

Fita da fada é feia

O saci saiu cedo

Chico chupa chupeta

Articulação:
$\square$ Normal $\square$ Fraca pressão
$\square$ Substituição compensatória
$\square$ Co-articulação compensatória $\square$ Distorção $\quad \square$ Distúrbio simples

$\square$ Outros:

Hipernasalidade:

$\square$ Ausente $\square$ Leve $\square$ Evidente

Nasofaringoscopia

Fibroscópio na narina:

$\square \mathrm{D} \quad \square \mathrm{E} \quad \square$ Meato médio $\square$ Meato inferior 
Esquema da velofaringe:

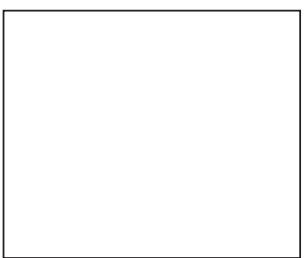

Em repouso

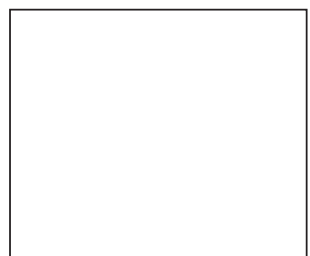

Melhor fechamento

Comportamento da velofaringe nas diversas atividades solicitadas:

Sopro:

la/:

$/ \mathrm{p} /:$

$/ \mathrm{t} / \mathrm{:}$

$/ \mathrm{k} / \mathrm{:}$

/f/:

$/ \mathrm{s} /:$

/S/:

Papai pediu pipoca:

O tatu tá na toca:

Cacá quer coca-cola:

Fita da fada é feia:

O saci saiu cedo:

Chico chupa chupeta:

Mobilidade de palato mole:

$\square$ Ausente $\square$ Pobre $\square$ Moderada $\square$ Boa

Mobilidade de paredes laterais da faringe:

$\square$ Ausente $\square$ Pobre $\square$ Moderada $\square$ Boa

Fechamento veloadenoideano:

$\square+\square$ -

\section{Gap predominantemente:}

$\square$ Ausente $\square$ Pequeno $\square$ Médio $\square$ Grande

$\square$ Coronal $\square$ Sagital $\square$ Circular $\square$ Circular com Prega de Passavant

Piora da função velofaríngea com maior complexidade da fala:

$\square+\square$ -

Melhora da função velofaríngea com treino articulatório:

$\square+\square$ -

Achados ORL:

Observações:

Conclusão:

Conduta:

Exame realizado por: ORL: 